\title{
Polymorphisms in folate-metabolizing genes, chromosome damage, and risk of Down syndrome in Italian women: identification of key factors using artificial neural networks
}

\author{
Fabio Coppedè ${ }^{1 *}$, Enzo Grossi ${ }^{2,3}$, Francesca Migheli $^{1}$, Lucia Migliore ${ }^{1}$
}

\begin{abstract}
Background: Studies in mothers of Down syndrome individuals (MDS) point to a role for polymorphisms in folate metabolic genes in increasing chromosome damage and maternal risk for a Down syndrome (DS) pregnancy, suggesting complex gene-gene interactions. This study aimed to analyze a dataset of genetic and cytogenetic data in an Italian group of MDS and mothers of healthy children (control mothers) to assess the predictive capacity of artificial neural networks assembled in TWIST system in distinguish consistently these two different conditions and to identify the variables expressing the maximal amount of relevant information to the condition of being mother of a DS child.

The dataset consisted of the following variables: the frequency of chromosome damage in peripheral lymphocytes (BNMN frequency) and the genotype for 7 common polymorphisms in folate metabolic genes (MTHFR 677C $>$ T and 1298A >C, MTRR 66A>G, MTR 2756A>G, RFC1 80G>A and TYMS 28bp repeats and $14946 \mathrm{bp}$ deletion). Data were analysed using TWIST system in combination with supervised artificial neural networks, and a semantic connectivity map.

Results: TWIST system selected 6 variables (BNMN frequency, MTHFR 677TT, RFC1 80AA, TYMS 1494 6bp +/+, TYMS 28bp 3R/3R and MTR 2756AA genotypes) that were subsequently used to discriminate between MDS and control mothers with $90 \%$ accuracy. The semantic connectivity map provided important information on the complex biological connections between the studied variables and the two conditions (being MDS or control mother).

Conclusions: Overall, the study suggests a link between polymorphisms in folate metabolic genes and DS risk in Italian women.
\end{abstract}

\section{Background}

Folates are essential nutrients required for one-carbon biosynthetic and epigenetic processes. After intestinal absorption, folate metabolism requires reduction and methylation into the liver to form 5-methyltetrahydrofolate (5-methylTHF), release into the blood and cellular uptake; then it can be used for the synthesis of DNA and RNA precursors or for the conversion of homocysteine (Hcy) to methionine, which is then used to form the main DNA methylating agent S-adenosylmethionine

\footnotetext{
* Correspondence: f.coppede@geog.unipi.it

'Department of Human and Environmental Sciences, Section of Medical Genetics, University of Pisa, Italy

Full list of author information is available at the end of the article
}

(SAM) $[1,2]$. A diagram illustrating folate metabolism is shown in figure 1. Deficiencies in cellular folates result in aberrant DNA methylation, point mutations, chromosome breakage, defective chromosome recombination and aneuploidy [3]. Therefore, in 1999 James and coworkers suggested that impairments in folate metabolism due to genetic polymorphisms of metabolic enzymes could increase the risk for having an infant with Down syndrome (DS) [4]. That paper stimulated considerable investigation into the possible role of folate metabolism in the risk of having a DS child and we recently reviewed all the genetic association studies performed from 1999 to 2009 [2]. Few additional papers have been produced after the publication of that review [5-8] for a 


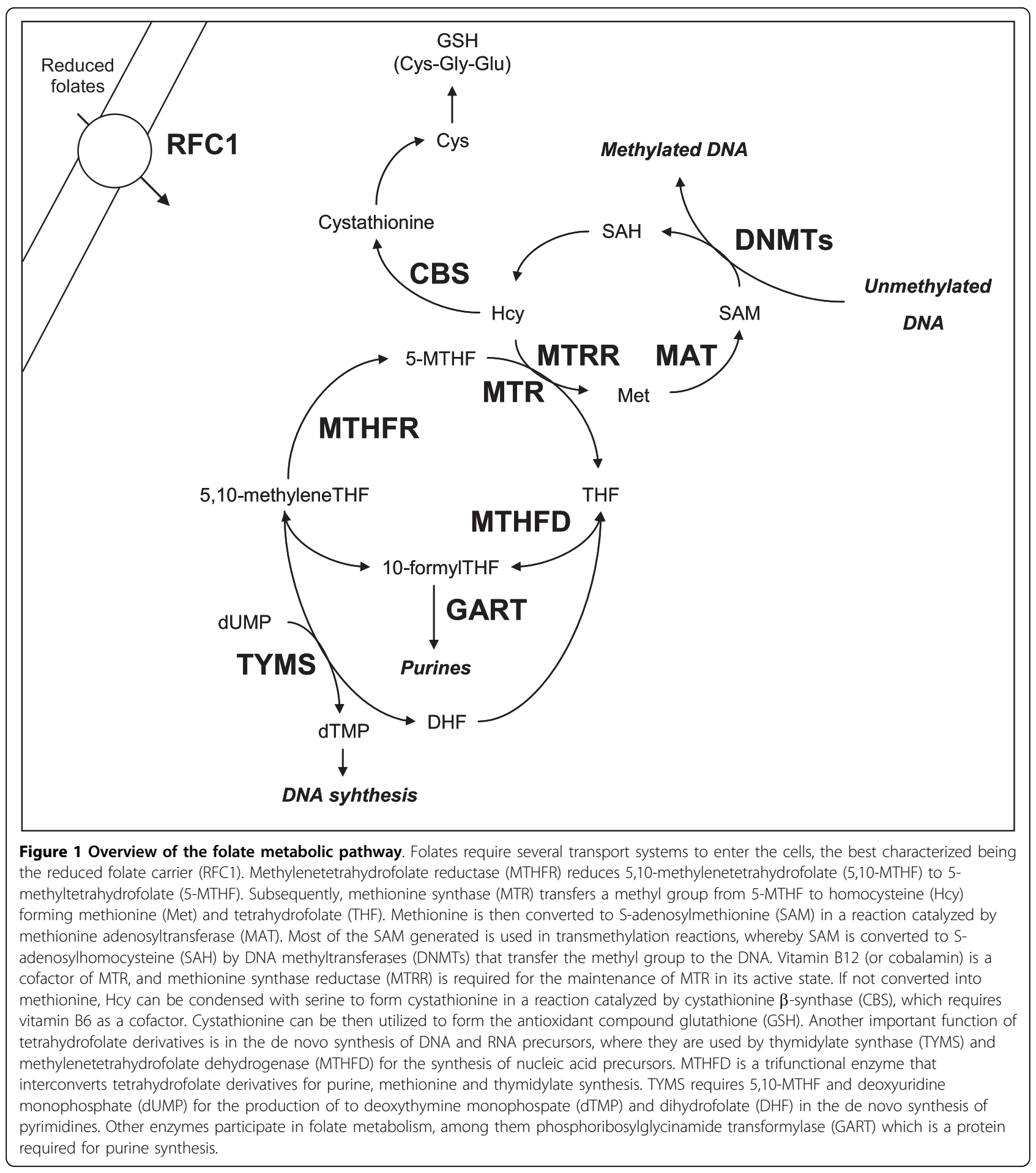

total of almost 30 research articles available in Pubmed and aimed at addressing this issue. However, despite considerable researches in the field, results are often conflicting or inconclusive and the question is still unsolved $[2,9,10]$. The current opinion is that the combined presence of several polymorphisms in the folate metabolic pathway in interaction with environmental factors might increase the risk for a DS pregnancy, rather than the presence of single polymorphic variants alone. However, the major limit of all the studies performed so far is the small sample size of the case-control groups which largely reduces the statistical power to test for genetic associations and for gene-gene and gene-environment interactions by means of traditional 
statistical approaches, such as logistic regression analyses [2]. Given the complexity of the folate metabolic pathway and the number of genes and environmental factors involved, we have estimated that the design of a case-control study able to test the contribution of each of these factors to DS risk with adequate power would require several thousands individuals [2]. Unfortunately, all the genetic association studies so far have been performed in groups of 100-200 mothers of DS individuals (MDS) or less, making it impossible to come to a definitive conclusion [2].

We observed that MDS who had a DS child in young age (35 years or before) have an increased frequency of chromosome damage and malsegregation events in peripheral blood lymphocytes, detectable by means of the micronucleus assay coupled by fluorescence in situ hybridization technique compared to women who gave birth to healthy children [11]. Others have recently suggested that women who have a DS child in young age might have an increased tendency to chromosome malsegregation events beginning from the first embryonic mitotic divisions of their body [12]. Subsequent studies by us revealed association between folate gene polymorphisms and the frequency of binucleated micronucleated lymphocytes (BNMN\%o) in blood from MDS and control mothers, providing an easily accessible biomarker (BNMN frequency) that can be measured to link impaired folate metabolism to chromosome malsegregation $[13,14]$.

Overall, genetic association studies as well as cytogenetic studies performed in MDS point to a possible role of folate gene polymorphisms in affecting chromosome malsegregation events, thus increasing the risk for a DS pregnancy. Moreover, they all suggest complex interactions between several factors within the folate metabolic pathway [2].

We performed the present study using Artificial Neural Networks (ANNs) to identify key factors linking folate metabolism to chromosome malsegregation and the risk of having a DS child. The method used by ANNs aims to understand natural processes and recreate those processes using automated models. These networks allow a method of forecasting with understanding of the relationship between variables, in particular nonlinear relationships [15-17]. ANNs function by initially learning a known set of data from a given problem with a known solution (training) and then the networks, inspired by the analytical processes of the human brain, are able to reconstruct the imprecise rules which may be underlying a complex set of data (testing). In recent years ANNs have been used successfully in medicine, for example they have been used to investigate the predictive values of risk factors on the conversion of amnestic mild cognitive impairment to Alzheimer's disease [18], to identify placental determinants of fetal growth [19], to identify genetic variants essential to differentiate sporadic amyotrophic lateral sclerosis cases from controls [20], and to distinguish between Alzheimer's disease patients and controls [21], among others.

This study aimed to analyze a dataset of genetic and cytogenetic data obtained from MDS and mothers of healthy children (control mothers) $[13,14]$ to assess the predictive capacity of artificial neural networks assembled in TWIST system [22] in distinguish consistently these two different conditions and to identify the variables expressing the maximal amount of relevant information to the condition of being mother of a DS child. A series of supervised multilayer perceptrons with four hidden units were then employed to validate the choice of variables made by TWIST system. Moreover, we constructed a semantic connectivity map to offer some insight regarding the complex biological connections between the studied variables and the two conditions (being MDS or control mother).

\section{Methods}

\section{Database}

We aimed to re-analyze from a completely new perspective most of the data obtained from our previous studies $[13,14]$. From a previously described database $[13,14]$ containing data from MDS (35 years or less; range 1935 ) that gave birth to a DS child and data from control mothers (35 years or less; range 20-35) that gave birth to healthy children and had no miscarriages or complications during pregnancies, we have selected 29 MDS and 32 control mothers for whom all the following information was available: 1) BNMN frequency, 2) genotype for the MTHFR $677 \mathrm{C}>\mathrm{T}$ polymorphism (CC, CT or TT), 3) genotype for the MTHFR $1298 \mathrm{~A}>\mathrm{C}$ polymorphism (AA, AC or CC), 4) genotype for the MTRR $66 \mathrm{~A}>\mathrm{G}$ polymorphism (AA, AG or GG), 5) genotype for the MTR 2756A $>$ G polymorphism (AA, AG or GG), 6) genotype for the RFC1 $80 \mathrm{G}>\mathrm{A}$ polymorphism (GG, AG, AA), 7) genotype for TYMS 28bp repeats (2R2R, 2R3R,3R3R) and 1494 6bp deletion $(+/+,+/-,-/-)$ polymorphisms. Table 1 shows the distribution of these variables among MDS and control mothers. As detailed elsewhere $[13,14]$ all MDS and control mothers included in the database were Caucasians of Italian origin (Tuscany and neighbouring areas). Concerning MDS, full trisomy 21 of the children (primary trisomy) was confirmed by cytogenetic analysis. Control mothers had no miscarriages or children affected by genetic disorders in their life, and at least one healthy child before age 35 years; they were recruited by us among women employed in the University Hospital of Pisa. All the subjects included in the study were matched for age either at time of delivery and at sampling (Table 2). The 
Table 1 Distribution of studied variables among MDS and control mothers

\begin{tabular}{|c|c|c|}
\hline Variable & MDS $(n=29)$ & Controls $(n=32)$ \\
\hline \multirow[t]{3}{*}{$\overline{M T H F R ~ 677 C>T}$} & CC: 5 & CC: 11 \\
\hline & CT: 19 & $\mathrm{CT}: 17$ \\
\hline & $\pi: 5$ & $\Pi: 4$ \\
\hline \multirow[t]{3}{*}{ MTHFR $1298 \mathrm{~A}>\mathrm{C}$} & AA: 14 & AA: 13 \\
\hline & AC: 15 & AC: 19 \\
\hline & CC: 0 & CC: 0 \\
\hline \multirow[t]{3}{*}{ MTRR $66 \mathrm{~A}>\mathrm{G}$} & AA: 9 & AA: 12 \\
\hline & AG: 14 & AG: 14 \\
\hline & GG: 6 & GG: 6 \\
\hline \multirow{3}{*}{ MTR 2756 A>G } & AA: 20 & AA: 24 \\
\hline & AG: 9 & AG: 6 \\
\hline & GG: 0 & GG: 2 \\
\hline \multirow[t]{3}{*}{$R F C 180 \mathrm{G}>\mathrm{A}$} & GG: 12 & GG: 10 \\
\hline & GA: 16 & GA: 12 \\
\hline & $\mathrm{AA}: 1$ & AA: 10 \\
\hline \multirow[t]{3}{*}{ TYMS 28bp repeat } & 2R/2R: 5 & 2R/2R: 6 \\
\hline & 2R/3R: 19 & 2R/3R: 14 \\
\hline & $3 R / 3 R: 5$ & 3R/3R: 12 \\
\hline \multirow[t]{3}{*}{ TYMS 1494 6bp deletion } & $6 \mathrm{bp}+/+: 5$ & $6 \mathrm{pb}+/+: 8$ \\
\hline & $6 b p+/-: 21$ & $6 b p+/-: 19$ \\
\hline & $6 b p-/-: 3$ & $6 b p-/-: 5$ \\
\hline BNMN\%o (mean \pm SD) & $16.5 \pm 7.6$ & $9.3 \pm 3.1$ \\
\hline
\end{tabular}

individuals included in the database have been selected after the administration of a detailed questionnaire, designed to document their previous conditions, dietary habits, smoking habits, working environment and pharmacological treatments in order to exclude those subjects exposed to environmental factors known to interfere with the BNMN frequency. Additional information can be found in our previous publications $[13,14]$.

The database analyzed in the present study is provided as additional material (see additional file 1: database, .csv

Table 2 Demographic characteristics of the study population

\begin{tabular}{llll}
\hline Study group & $\begin{array}{l}\text { Number of } \\
\text { subjects }\end{array}$ & $\begin{array}{l}\text { Age at } \\
\text { delivery } \\
\text { mean } \pm \text { S.D. }\end{array}$ & $\begin{array}{l}\text { Age at } \\
\text { sampling } \\
\text { mean } \pm \text { S.D. }\end{array}$ \\
\hline MDS & 29 & $28.2 \pm 4.7$ & $49.6 \pm 11.2$ \\
\hline $\begin{array}{l}\text { Control } \\
\text { mothers }\end{array}$ & 32 & $28.5 \pm 5.2$ & $47.3 \pm 6.9$ \\
\hline
\end{tabular}

\begin{tabular}{|l|l|r|r|r|}
\hline record & variant & MTRR66_AA & MTRR66_AG & MTRR66_GG \\
\hline record 1 & MTRR66_AA & 1 & 0 & 0 \\
\hline record 2 & MTRR66_GG & 0 & 0 & 1 \\
\hline record 3 & MTRR66_AG & 0 & 1 & 0 \\
\hline
\end{tabular}

Figure 2 Method of coding the polymorphisms in the database. The code assigned to the polymorphisms transformed each polymorphism in three genotype classes: major homozygous, heterozygous and minor homozygous. For each class a binary coding was applied: 0 if variable absent; 1 if variable present. So for example considering the polymorphism MTRR $66 \mathrm{~A}>\mathrm{G}$ which can exist in three variants: AA (major homozygous), AG (heterozygous) and $G G$ (minor homozygous). Supposing that three records are AA, $G G$ and $A G$, the coding has been applied as shown in the figure.

format). Figure 2 explains how genotypes were coded in the database.

\section{Genotyping and BNMN frequency}

The database data concerning the BNMN frequency and the genotype for all the 7 studied polymorphisms had been previously obtained by means of the cytokinesisblock micronucleus assay (BNMN frequency) and validated PCR/RFLP techniques as described elsewhere $[13,14]$. All the samples were coded and data were processed by blinded operators. All individuals gave informed consent for inclusion in the database, whose creation was performed in accordance with the Helsinki Declaration and approved by the "Stella Maris" I.R.C.C. S. Ethics Committee as described elsewhere $[13,14]$.

\section{Artificial neural networks analysis}

Advanced intelligent systems based on novel coupling of artificial neural networks and evolutionary algorithms have been applied. In this study we applied TWIST system [22] and supervised ANNs in order to develop a model able to predict with high degree of accuracy the diagnostic class starting from genotype data alone. Supervised ANNs are networks which learn by examples, calculating an error function during the training phase and adjusting the connection strengths in order to minimize the error function [23]. The learning constraint of the supervised ANNs makes their own output coincide with the predefined target. The general form of these ANNs is: $y=f\left(x, w^{*}\right)$, where $w^{*}$ constitutes the set of parameters which best approximate the function.

\section{TWIST system}

Data analysis was performed using a re-sampling system named TWIST developed by Semeion Research Centre. The TWIST system consists in an ensemble of two previously described systems: T\&T and IS [22]. The T\&T system is a robust data re-sampling technique that is able to arrange the source sample into sub-samples that all possess a similar probability density function. In this way, the data is split into two or more sub-samples in 
order to train, test and validate the ANN models more effectively. The IS system is an evolutionary wrapper system able to reduce the amount of data while conserving the largest amount of information available in the dataset. The combined action of these two systems allow us to solve two frequent problems in managing Artificial Neural Networks, i.e. the optimal splitting of the data set in training and testing subsets containing a balanced distribution of outliers and the optimal selection of variables with maximal amount of information relevant to the problem under investigation. Both systems are based on a Genetic Algorithm, the Genetic Doping Algorithm (GenD) developed at Semeion Research Centre [24]. The TWIST system has been previously applied in different medical contexts [25], additional data are given (see additional file 2: Twist System, pdf file).

After this processing, the features that were most significant for the classification were selected and at the same time the training set and the testing set were created with a function of probability distribution similar to the one that provided the best results in the classification. A series of supervised Multi Layer Perceptrons, with four hidden units, were then used for the classification task. The final ANNs which were trained and tested on the new data set generated by TWIST system are "virgin" and operate independently and blindly from each other and from TWIST system.

\section{Semantic connectivity map}

An existing mapping method [26,27] was used to highlight through a graph the most important links among variables, using a mathematical approach based on an artificial adaptive system called Auto Contractive MapAuto-CM algorithm. The Auto Contractive Map (Auto$\mathrm{CM}$ ) is a special kind of Artificial Neural Network able to find, by a specific data mining learning algorithm, the consistent patterns and/or systematic relationships and hidden trends and associations among variables. After the training phase the weights developed by Auto-CM are proportional to the strength of associations of all variables each-other. The weights are then transformed in physical distances. Variables couples whose connection weights are higher become nearer and vice versa. A simple mathematical filter represented by minimum spanning tree is applied to the distances matrix and a graph is generated. This allows seeing connection schemes among variables and detecting variables acting ad "hubs", being highly connected. This matrix of connections preserves non linear associations among variables and captures connection schemes among clusters.

After the training phase, the weights matrix of the Auto-CM represents the warped landscape of the dataset. Subsequently, a simple filter to the weights matrix of the Auto-CM system was applied to obtain a map of the main connections between the variables of the dataset and the basic semantic of their similarities, defined connectivity map as detailed by Buscema and Grossi [26] and Buscema et al. [27]. The theory behind Auto$\mathrm{CM}$ system is provided as additional material (see additional file 3: Auto-CM System, pdf file).

\section{Results}

\section{Classification performances with ANNs}

The linear correlation index between the input variables and the target variable was generally very low, with exception of BNMN\%o $(r=0.54)$. This gave the rationale to employ artificial neural networks.

The application of TWIST system allowed the selection of a subgroup of 6 variables described in table 3 . This new data set has been analyzed with Back propagation ANNs employing a rigorous validation protocol. The validation protocol is a procedure to verify the models' ability to generalize the results reached in the testing phase. Among the different protocols reported in literature, the selected model is the protocol with the greatest generalization ability on data unknown to the model itself. The procedural steps in developing the validation protocol are: 1) subdividing the dataset randomly into two sub-samples: the first called Training Set, and the second, called Testing Set; 2) choosing a fixed ANN (and/or Organism) which is trained on the Training Set. In this phase, the ANNs learns to associate the input variables with those that are indicated as targets; 3 ) saving the weight matrix produced by the ANNs at the end of the training phase, and freezing it with all of the parameters used for the training; 4) showing the Testing Set to the ANNs, so that in each case, the ANNs can express an evaluation based on the training just performed. This procedure takes place for each input vector but every result (output vector) is not communicated to the ANNs; in this way, the ANNs are evaluated only in reference to the generalization ability that it has acquired during the Training phase; 5) constructing a new ANN with identical architecture to the previous one and repeating the procedure from point 1 . This general training plan has been employed five times with obtaining 10 independent classification

\section{Table 3 Variables selected by TWIST system}

\begin{tabular}{ll}
\hline & Selected Variables \\
\hline 1 & MTR 2756 AA \\
2 & MTHFR 677TT \\
3 & RFC1 80 AA \\
4 & TYMS 1494 6bp +/+ \\
5 & TYMS 28bp 3R/3R \\
6 & BNMN \%o \\
\hline
\end{tabular}


experiments. We have employed the so called $5 \times 2$ crossvalidation protocol [28] which produces 2 elaborations for every sample, the first with training on subset a and testing on subset $b$ and the second with training on subset $b$ and testing on subset a. Table 4 summarizes the results obtained with back propagation ANN applied ten times on the final data set. Figure 3 shows the Area Under the Curve (AUC) of Receiver-Operating Characteristic (ROC) AUC of the ten classifications and the average ROC AUC in red.

\section{Semantic connectivity map}

Figure 4 shows the semantic connectivity map obtained with the application of Auto-CM system. Variables which have the maximal amount of connections with other variables are called "hubs" of the system. In order to better understand the meaning of the connections a numerical value is applied to each edge of the graph. This value, deriving from the original weight developed by Auto-CM during the training phase scaled from 0 to 1 , is proportional to the strength of the connections among two variables.

The TYMS $14946 \mathrm{bp}+/$ - genotype resulted to be the principal hub of the system, i.e. the dominant variable. This variable was connected with $T Y M S 28 \mathrm{bp} 2 \mathrm{R} / 3 \mathrm{R}$, with MTR 2756AA, and with both MTHFR 677CT and MTHFR 1298AA variables. Connections were detected among MTHFR 677 and 1298 variables; particularly, MTHFR 677TT was connected with MTHFR 1298AA, whereas MTHFR $1298 \mathrm{AC}$ was connected with both

Table 4 Classification performances of back propagation neural networks on final data set

\begin{tabular}{lllll}
\hline ANN & Sensitivity & Specificity & $\begin{array}{l}\text { Global } \\
\text { accuracy }\end{array}$ & $\begin{array}{l}\text { ROC } \\
\text { AUC }\end{array}$ \\
\hline $\begin{array}{l}\text { Back propagation 1 } \\
\text { (a-b) }\end{array}$ & 80 & 93,33 & 86,67 & 0,864 \\
$\begin{array}{l}\text { Back propagation 2 } \\
\text { (a-b) }\end{array}$ & 80 & 93,33 & 86,67 & 0,851 \\
$\begin{array}{l}\text { Back propagation 3 } \\
\text { (a-b) }\end{array}$ & 80 & 93,33 & 86,67 & 0,864 \\
$\begin{array}{l}\text { Back propagation 4 } \\
\text { (a-b) }\end{array}$ & 80 & 93,33 & 86,67 & 0,916 \\
$\begin{array}{l}\text { Back propagation 5 } \\
\text { (a-b) }\end{array}$ & 86,67 & 93,33 & 90 & 0,882 \\
$\begin{array}{l}\text { Back propagation 1 } \\
\text { (b-a) }\end{array}$ & 100 & 94,12 & 97,06 & 0,958 \\
$\begin{array}{l}\text { Back propagation 2 } \\
\text { (b-a) }\end{array}$ & 100 & 88,24 & 94,12 & 0,956 \\
$\begin{array}{l}\text { Back propagation 3 } \\
\text { (b-a) }\end{array}$ & 100 & 88,24 & 94,12 & 0,945 \\
$\begin{array}{l}\text { Back propagation 4 } \\
\text { (b-a) }\end{array}$ & 92,86 & 94,12 & 93,49 & 0,926 \\
$\begin{array}{l}\text { Back propagation 5 } \\
\text { (b-a) }\end{array}$ & 100 & 94,12 & 97,06 & $\mathbf{0 , 9 1}$ \\
\begin{tabular}{l} 
Mean \\
\hline
\end{tabular} & $\mathbf{8 9 , 9 5}$ & $\mathbf{9 2 , 5 5}$ & $\mathbf{9 1 , 2 5}$ & \\
\hline
\end{tabular}

MTHFR $677 C C$ and MTHFR $677 C$ T variables. Moreover, TYMS 28bp repeats and 1494 6bp deletion variables were always connected each other; particularly, TYMS $14946 \mathrm{bp}+/$ - resulted connected with TYMS 28bp 2R/3R, TYMS 1494 6bp +/+ with TYMS 28bp 2R/ 2R, and TYMS $14946 \mathrm{bp}-/$ - with TYMS 28bp 3R/3R. BNMN\%, RFC1 80GG and TYMS 28bp 2R/3R resulted to be the three variables connected with the condition of being MDS. On the contrary, RFC1 80AA, MTR 2756AA and TYMS 28bp 3R/3R resulted to be the three variables connected with the condition of being a control mother.

\section{Discussion}

This study explored the association between 7 polymorphisms in the folate metabolic pathway, chromosome damage in peripheral blood lymphocytes (as BNMN frequency), and the condition of being mother of a DS child with complementary non-linear approaches: supervised neural networks (ANNs), and the semantic connectivity map.

Through TWIST system, we established a consistent possibility to predict the status of being a MDS on the basis of 6 selected variables (Table 3) with 90\% specificity, sensitivity and global accuracy (Table 4), this meaning that the selected variables contained specific information on the occurrence of a DS pregnancy. In particular, the BNMN frequency, as well as RFC1 80AA, MTHFR 677TT, MTR 2756AA, TYMS 1494 6bp del +/+ and TYMS $28 \mathrm{bp} 3 \mathrm{R} / 3 \mathrm{R}$ polymorphisms resulted the most important variables for discriminating between MDS and control mothers (Table 3). Most of these variables had been previously associated with DS risk by means of genetic association studies [2].

The present study represents the first attempt to use ANNs to understand the complex relationship between folate metabolism and maternal risk for having a DS child. Though we achieved good results using ANNs for a small dataset, results are not necessarily generalizable to a larger population but need to be validated independently in future studies. A look at Table 1 shows that some of the genotypes, such as for example the MTHFR $1298 \mathrm{CC}$ one, were not present in our cohort while others were present only in a few subjects, indicating the need of further analyses in a larger group. Within this context we made our database available online (see additional file 1) so that other research groups can use the data to increase their own datasets. Moreover, we welcome any further analysis of our database with other methods of forecasting, including logistic regression methods, neural networks, or support vector machines. In addition, present results have been obtained in an Italian case-control cohort and are not generalizable to other populations. 


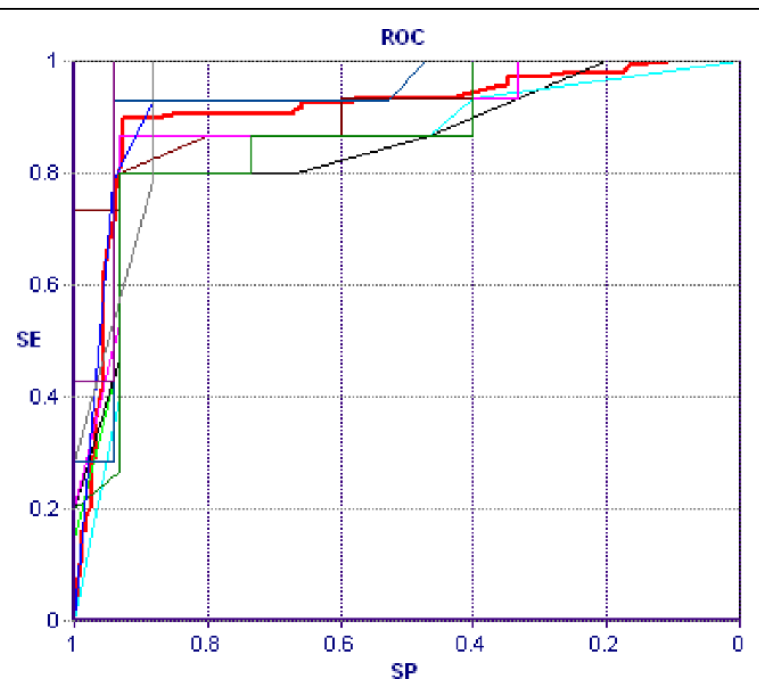

- THavroc[auc 0.91]

- FF_Ep 4 baAUTO(5)[AUUC 0.958]

- FF BP 4 ABAUTO(1)[AUC 0.851]

- FF_Bp 4 ABA.UTO(2)[A.UC 0.864]

- FF_Bp 4 ABA.UTO(3)[A.UC 0.916]

- FF Bp 4 ABAUTO(4)][AUC 0.882]

- FF_Ep 4 ABAUTO(5)][AUUC 0.864]

- FF_BP 4 BA.AUTO(1)[AUC 0.956]

-FF_Bp 4 BA.AUTO(2)[AUC 0.945]

- FF Bp 4 BA.AUTO(3)[AULC 0.926]

- FF_Bp 4 baAUTTO(4)[AMUC 0.966]

Figure 3 Area Under the Curve (AUC) of Receiver Operator Characteristic (ROC) relative to ten classification tasks performed with Back Propagation ANN models. The ROC represents therelationship between sensitivity and specificity for the prediction of each of the considered outcomes. The average ROC is depicted in red

Through the connectivity map (Figure 4 ) we established the connections between the studied variables and the condition of being MDS or control mother.

Considering individual findings the connectivity map showed several connections already known in the literature, as well as novel ones. Particularly, a connection resulted between MTHFR $677 \mathrm{C}>\mathrm{T}$ and $1298 \mathrm{~A}>\mathrm{C}$ polymorphisms, for example among the MTHFR 677TT and the MTHFR 1298AA genotypes. This connection can be explained on the basis of a strong linkage disequilibrium existing between the two polymorphisms. MTHFR works as a dimer protein and the combined presence of

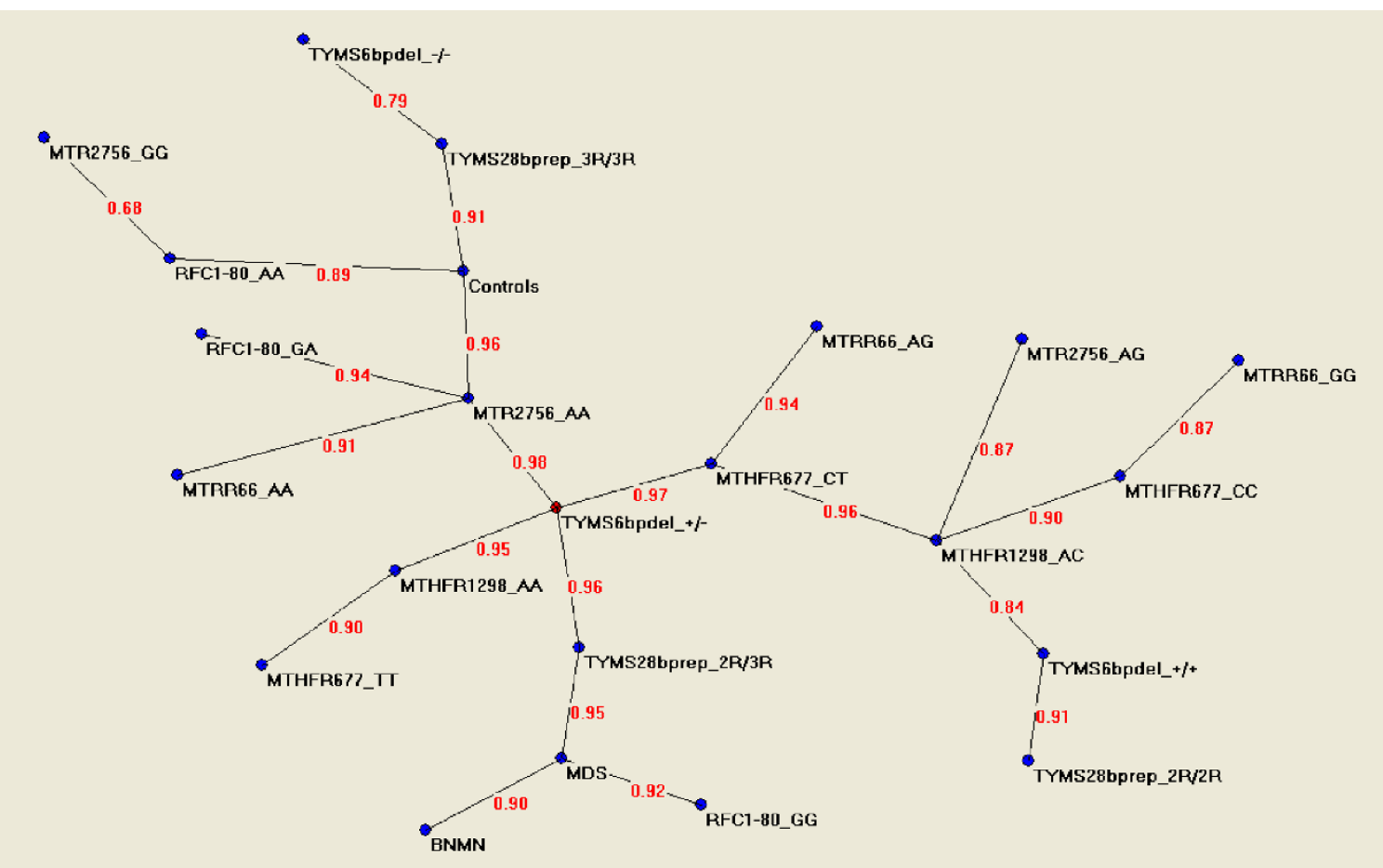

Figure 4 Semantic connectivity map obtained with Auto- $\mathrm{Cm}$ System. The figures on the arches of the graph refer to the strength of the association Between two adjacent nodes. The range of this value is from 0 to 1. 
the $\mathrm{T}$ allele at position 677 and of the $\mathrm{C}$ allele at position 1298 impairs the stability of the dimer. As a consequence, the $677 \mathrm{~T}$ allele is in strong linkage with the $1298 \mathrm{~A}$ one, while the $677 \mathrm{~T}-1298 \mathrm{C}$ haplotype is rare and selected negatively [29].

Similarly, the connectivity map (Figure 4) showed a connection between TYMS 28bp repeats and 1494 6bp deletion polymorphisms. For example, the TYMS 1494 $6 \mathrm{bp}+/+$ genotype resulted connected with the TYMS 28bp 2R/2R one, and the TYMS 1494 6bp -/- genotype with the TYMS 28bp 3R/3R one. Again, linkage disequilibrium is known between these two polymorphisms. A previous study by us, performed in a large cohort of white non-Hispanic Americans, revealed that haplotypes containg both the $6 \mathrm{bp}$ deleted (-) allele and the $2 \mathrm{R}$ allele are rare [30]. A possible biological explanation could be that the 6bp deletion is likely to impair the stability of the TYMS mRNA, while the $2 \mathrm{R}$ allele is associated with reduced transcription of the TYMS gene. Therefore, their combined presence on the same haplotype could seriously impair TYMS production [30].

The connectivity map (Figure 4) revealed that three variables are closely connected with the condition of being MDS: the RFC1 $80 \mathrm{GG}$ genotype, the BNMN frequency, and the TYMS 2R/3R genotype. On the contrary, the RFC1 80AA genotype, the TYMS 3R/3R genotype and the MTR 2756AA genotype, are closely connected to the condition of being a control mother. The association between the RFC1 80G allele and increased risk for having a DS child has been often observed in Italian populations. We first reported a possible role for the RFC1 $80 \mathrm{G}>\mathrm{A}$ polymorphism, in interaction with MTHFR $677 \mathrm{C}>\mathrm{T}$ and $1298 \mathrm{~A}>\mathrm{C}$ variants, in affecting DS risk in Italy, suggesting a causative role for the RFC1 $80 \mathrm{G}$ allele and a protective role for the RFC1 $80 \mathrm{~A}$ one [31]. Subsequently, in a larger case-control study, others observed an independent association between the RFC1 80GG genotype and increased risk for having a DS child in Italy [32]. They also confirmed the protective and interactive role for the RFC1 80A allele previously observed by us [33]. Overall, based on these studies, we concluded that in the Italian population the RFC1 $80 \mathrm{G}$ allele could increase the risk for having a DS child, while the RFC1 $80 \mathrm{~A}$ allele could be protective [34]. Interestingly, the connectivity map confirmed this observation, showing a connection between the RFC1 $80 \mathrm{GG}$ genotype and the condition of being MDS, as well as a connection between the RFC1 80AA genotype and the condition of being control mother.

The BNMN frequency is another variable connected with the condition of being MDS. Several previous studies by us $[11,13,14]$ revealed a statistically significant increased BNMN frequency in MDS respect to control mothers, leading us to formulate the hypothesis that
MDS could have an increased tendency to chromosome malsegregation events during somatic mitotic divisions. More recently others have suggested that women who have a DS child in young age could be characterized by an elevated frequency of mitotic malsegregation events during embryogenesis [12].

The MTR 2756AA genotype resulted to be connected to the condition of being a control mother as well as to the MTRR 66AA genotype (Figure 4). As shown in figure 1, MTR and MTRR physically interact during folate metabolism, being MTRR required for the maintenance of MTR in its active state. In 2003, Bosco and co-workers observed association between the MTR 2756G allele and increased DS risk in Italy, arguing for a protective role for the 2756A allele. They also reported an interaction between MTR 2756A $>\mathrm{G}$ and MTRR 66A $>\mathrm{G}$ polymorphisms in increasing DS risk [35]. We recently observed interactions between MTR 2756AA and MTHFR 677TT genotypes in increasing DS risk in Italy [14]. However, as shown by the connectivity map (Figure 4), these two variables are not directly connected, and many other factors might affect their interaction.

A very interesting finding of the present study is in the central role played by TYMS polymorphisms in the connectivity map. Indeed, the TYMS $6 \mathrm{bp}+/$ - genotype resulted to be the principal hub of the system, the TYMS 28bp 2R/3R genotype was connected to the condition of being MDS, and the TYMS 28bp 3R/3R genotype to that of being a control mother (Figure 4). TYMS shifts the folate metabolic pathway from DNA methylation toward DNA synthesis (Figure 1). Both TYMS and MTHFR compete for the same substrate: 5,10-methyleneTHF, the first for DNA synthesis, the latter for DNA methylation purposes. The connectivity map (Figure 4) shows several connections between TYMS and MTHFR polymorphisms that can be explained by the following observation: given that both enzymes utilize the same substrate, polymorphisms reducing MTHFR enzyme activity might shift pools of 5,10-methyleneTHF from DNA methylation toward DNA synthesis, whereas polymorphisms affecting TYMS activity might shift the pathway from DNA synthesis toward DNA methylation [30]. We recently observed interaction between MTHFR and TYMS polymorphisms in increasing DS risk, suggesting that an impaired balance between DNA synthesis and methylation processes could favour chromosome malsegregation events [14]. The results from the present study (Table 3 and Figure 4) seriously argue in favour of a pivotal role for TYMS polymorphisms in DS risk.

\section{Conclusions}

In conclusion, the currently available literature suggests that complex interactions between polymorphisms in 
folate metabolizing genes might account for an increased maternal risk to have a DS child; however, given the complexity of the pathway, those complex interactions cannot be easily understood and none of the so far studied polymorphisms can be used in genetic counselling to predict the maternal risk for having a DS child [2]. The present study identified 6 critical variables that allowed discriminating between MDS and control mothers with $90 \%$ sensitivity, specificity and accuracy, and provided important information on the complex biological connections between the studied variables and the two conditions (being MDS or control mothers). The study suggests a link between polymorphisms in the folate metabolic pathway and the risk for a DS pregnancy, indicating complex gene-gene interactions. The overall evidence suggests that further research in the field, such as the addition of other variables, is likely to increase the specificity and sensitivity of the system and could help for the development of screening tools aimed at evaluating the risk for a young woman to have a DS pregnancy.

\section{Additional material}

Additional file 1: Database. The database used for analysis in an Excel CSV format.

Additional file 2: Twist System. The pdf file contains a detailed description of the Twist system. This document already existed as documentation $[22,24,25]$ and is included for clarity.

Additional file 3: Auto-CM System. The pfd file contains a detailed description of the theory behind Auto-CM system. This document already existed as documentation $[26,27]$ and is included for clarity.

\section{Acknowledgements}

Authors acknowledge MDS and control mothers for their kind participation in the study.

\section{Author details}

'Department of Human and Environmental Sciences, Section of Medical Genetics, University of Pisa, Italy. ${ }^{2}$ Bracco Medical Department, San Donato Milanese, Italy. ${ }^{3}$ Semeion Research Centre, Rome, Italy.

\section{Authors' contributions \\ FC: conceived and designed the study, supervised all the work related with the collection of genetic and cytogenetic data and all the work in the laboratory, prepared the database for the specific analysis, interpreted the data, wrote the manuscript. EG: carried out the ANNs and PC analysis, was involved with the drafting of the related technical parts in the manuscript including tables and figures, and revised critically the entire manuscript. FM: performed genotyping and helped preparing the database for the specific analysis. LM: supervised the research group, helped drafting and revising critically the entire manuscript. All authors read and approved the final manuscript.}

\section{Competing interests}

The authors declare that they have no competing interests.

Received: 26 March 2010 Accepted: 24 September 2010 Published: 24 September 2010
References

1. Bailey LB, Gregory JF: Folate metabolism and requirements. J Nutr 1999, 129:779-82.

2. Coppedè F: The complex relationship between folate/homocysteine metabolism and risk of Down syndrome. Mutat Res 2009, 682:54-70.

3. Fenech M: The role of folic acid and Vitamin B12 in genomic stability of human cells. Mutat Res 2001, 475:57-67.

4. James SJ, Pogribna M, Pogribny IP, Melnyk S, Hine RJ, Gibson JB, Yi P, Tafoya DL, Swenson DH, Wilson VL, Gaylor DW: Abnormal folate metabolism and mutation in the methylenetetrahydrofolate reductase gene may be maternal risk factors for Down syndrome. Am J Clin Nutr 1999, 70:495-501.

5. Biselli JM, Machado FB, Zampieri BL, Alves da Silva AF, Goloni-Bertollo EM,

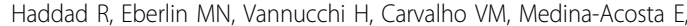
Pavarino-Bertelli EC: Double aneuploidy $(48, X X Y,+21)$ of maternal origin in a child born to a 13-year-old mother: evaluation of the maternal folate metabolism. Genet Couns 2009, 20:225-34.

6. Brandalize AP, Bandinelli E, dos Santos PA, Roisenberg I, Schüler-Faccini L: Evaluation of C677T and A1298C polymorphisms of the MTHFR gene as maternal risk factors for Down syndrome and congenital heart defects. Am J Med Genet A 2009, 149A:2080-7.

7. Fintelman-Rodrigues N, Corrêa JC, Santos JM, Pimentel MM, SantosRebouças CB: Investigation of CBS, MTR, RFC-1 and TC polymorphisms as maternal risk factors for Down syndrome. Dis Markers 2009, 26:155-61.

8. Kokotas H, Grigoriadou M, Mikkelsen M, Giannoulia-Karantana A Petersen MB: Investigating the impact of the Down syndrome related common MTHFR 677 C $>$ T polymorphism in the Danish population. Dis Markers 2009, 27:279-85.

9. Patterson D: Folate metabolism and the risk of Down syndrome. Downs Syndr Res Pract 2008, 12:93-7.

10. Zintzaras E: Maternal gene polymorphisms involved in folate metabolism and risk of Down syndrome offspring: a meta-analysis. J Hum Genet 2007, 52:943-53.

11. Migliore L, Boni G, Bernardini R, Trippi F, Colognato R, Fontana I, Coppedè F, Sbrana I: Susceptibility to chromosome malsegregation in lymphocytes of women who had a Down syndrome child in young age. Neurobiol Aging 2006, 27:710-6.

12. Hultén MA, Patel $\mathrm{S}$, Jonasson J, Iwarsson E: On the origin of the maternal age effect in trisomy 21 Down syndrome: the Oocyte Mosaicism Selection model. Reproduction 2010, 139:1-9.

13. Coppedè F, Colognato R, Bonelli A, Astrea G, Bargagna S, Siciliano G, Migliore L: Polymorphisms in folate and homocysteine metabolizing genes and chromosome damage in mothers of Down syndrome children. Am J Med Genet A 2007, 143A:2006-15

14. Coppedè F, Migheli F, Bargagna S, Siciliano G, Antonucci I, Stuppia L Palka G, Migliore L: Association of maternal polymorphisms in folate metabolizing genes with chromosome damage and risk of Down syndrome offspring. Neurosci Lett 2009, 449:15-9.

15. Penco S, Grossi E, Cheng S, Intraligi M, Maurelli G, Patrosso MC, Marocchi A, Buscema M: Assessment of the Role of Genetic Polymorphism in Venous Thrombosis Through Artificial Neural Networks. Annals of Human Genetics 2005, 69:693-706.

16. Lisboa PJC: A review of evidence of health benefit from artificial neural networks in medical intervention. Neural Networks 2002, 15:11-39.

17. Grossi E, Mancini A, Buscema M: International experience on the use of artificial neural networks in gastroenterology. Dig Liver Dis 2007, 39:278-85

18. Tabaton M, Odetti P, Cammarata S, Borghi R, Monacelli F, Caltagirone C, Bossù P, Buscema M, Grossi E: Artificial Neural Networks Identify the Predictive Values of Risk Factors on the Conversion of Amnestic Mild Cognitive Impairment. J Alzheimers Dis 2010, 19:1035-40.

19. Street ME, Grossi E, Volta C, Faleschini E, Bernasconi S: Placental determinants of fetal growth: identification of key factors in the insulinlike growth factor and cytokine systems using artificial neural networks. BMC Pediatr 2008, 8:24

20. Penco S, Buscema M, Patrosso MC, Marocchi A, Grossi E: New application of intelligent agents in sporadic amyotrophic lateral sclerosis identifies unexpected specific genetic background. BMC Bioinformatics 2008, 9:254

21. Grossi E, Buscema MP, Snowdon D, Antuono P: Neuropathological findings processed by artificial neural networks (ANNs) can perfectly 
distinguish Alzheimer's patients from controls in the Nun Study. BMC Neurol 2007, 7:15.

22. Buscema M, Grossi E, Intraligi M, Garbagna N, Andriulli A, Breda M: An optimized experimental protocol based on neuro-evolutionary algorithms application to the classification of dyspeptic patients and to the prediction of the effectiveness of their treatment. Artif Intell Med 2005, 34:279-305.

23. Rumelhart DE, Parallel JLMC Clelland: Distributed Processing: Explorations in the Microstructure of Cognition Cambridge, MA: Foundations, MIT Press 1986, 1.

24. Buscema M: Genetic Doping Algorihm (GenD): theory and applications. Expert Systems 2004, 21:63-79.

25. Buscema M, Capriotti M, Bergami F, Babiloni C, Rossini P, Grossi E: The implicit function as squashing time model: a novel parallel nonlinear EEG analysis technique distinguishing mild cognitive impairment and Alzheimer's disease subjects with high degree of accuracy. Comput Intell Neurosci 2007, 35021:1-15.

26. Buscema M, Grossi E: The semantic connectivity map: an adapting selforganising knowledge discovery method in data bases. Experience in gastro-oesophageal reflux disease. Int J Data Min Bioinform 2008, 2:362-404

27. Buscema M, Grossi E, Snowdon D, Antuono P: Auto-Contractive Maps: an artificial adaptive system for data mining. An application to Alzheimer disease. Curr Alzheimer Res 2008, 5:481-98.

28. Dietterich TG: Approximate Statistical Tests for Comparing Supervised Classification Learning Algorithms. Neural Comput 1998, 10:1895-1923.

29. Yamada K, Chen Z, Rozen R, Matthews RG: Effects of common polymorphisms on the properties of recombinant human methylenetetrahydrofolate reductase. Proc Natl Acad Sci USA 2001, 98:14853-14858.

30. Skibola CF, Forrest MS, Coppedè F, Agana L, Hubbard A, Smith MT, Bracci PM, Holly EA: Polymorphisms and haplotypes in folatemetabolizing genes and risk of non-Hodgkin lymphoma. Blood 2004, 104:2155-2162.

31. Coppedè F, Marini G, Bargagna S, Stuppia L, Minichilli F, Fontana I, Colognato R, Astrea G, Palka G, Migliore L: Folate gene polymorphisms and the risk of Down syndrome pregnancies in young Italian women. Am J Med Genet A 2006, 140:1083-1091.

32. Scala I, Granese B, Sellitto M, Salomè S, Sammartino A, Pepe A, Mastroiacovo P, Sebastio G, Andria G: Analysis of seven maternal polymorphisms of genes involved in homocysteine/folate metabolism and risk of Down syndrome offspring. Genet Med 2006, 8:409-416.

33. Scala I, Granese B, Lisi A, Mastroiacovo P, Andria G: Response to "folate gene polymorphisms and the risk of Down syndrome pregnancies in young Italian women" by F. Coppedè et al. (2006). Am J Med Genet A 2007, 143:1015-1017.

34. Coppedè F, Colognato R, Migliore L: MTHFR and RFC-1 gene polymorphisms and the risk of Down syndrome in Italy. Am J Med Genet A 2007, 143:1018-1019.

35. Bosco P, Gueant-Rodriguez RM, Anello G, Barone C, Namour F, Caraci F, Roman A, Romano C, Gueant JL: Methionine synthase (MTR) $2756(A>G)$ polymorphism, double heterozygosity methionine synthase $2756 \mathrm{AG} /$ methionine synthase reductase (MTRR) 66 AG, and elevated homocysteinemia are three risk factors for having a child with Down syndrome. Am J Med Genet A 2003, 121:219-224.

\section{Pre-publication history}

The pre-publication history for this paper can be accessed here: http://www.biomedcentral.com/1755-8794/3/42/prepub

doi:10.1186/1755-8794-3-42

Cite this article as: Coppedè et al:: Polymorphisms in folate-

metabolizing genes, chromosome damage, and risk of Down syndrome in Italian women: identification of key factors using artificial neural networks. BMC Medical Genomics 2010 3:42.

\section{Submit your next manuscript to BioMed Central and take full advantage of:}

- Convenient online submission

- Thorough peer review

- No space constraints or color figure charges

- Immediate publication on acceptance

- Inclusion in PubMed, CAS, Scopus and Google Scholar

- Research which is freely available for redistribution

Submit your manuscript at www.biomedcentral.com/submit
Ciomed Central 